\section{Slip and Twinning in Single Crystals of Mercury}

IN 1935 Prof. E. N. da C. Andrade and Mr. P. J. Hutchings described ${ }^{1}$ the results of interesting experiments on the deformation of single crystals of mercury, and it is concluded that in a mercury crystal the rhombohedral faces are the glide planes-as (it was stated) would be expected from the fact that these are the planes of closest packing. Of many measurements that had been made of the intersection angles between glide and twinning plane traces, three sets in particular were considered to furnish strong confirmation of the above conclusion.

I have recently had occasion to examine this analysis, and, as a result, would make the following observations :

Not the rhombohedral faces $\{100\}$ but the pyramidal planes $\{11 \overline{1}\}$ are those most closely packed in the mercury crystal lattice. This is readily seen on laying out the atomic positions, as in the accompanying diagram, in which the $\{100\}$ packing is shown at $(a)$ and the $\{11 \overline{1}\}$ packing at $(b)$. It will be seen that while the $\{100\}$ planes contain one line of close packing - the short diagonal of a rhomb face - the three $\{1 \mathrm{i} \bar{i}\}$ planes each contain the two short diagonals of adjacent rhomb faces, and therefore must be more closely packed than the $\{100\}$ planes.

This fact necessitates a reconsideration of the main conclusion of Andrade and Hutchings' analysisthat the rhomb faces are the glide planes. The position is, that if the $\{100\}$ planes are the glide planes, as appeared to be indicated by the recorded angular measurements, which fulfil the geometrical requirements, then glide in mercury crystals does not follow the general rule for face-centred metals, namely, that it takes place on the most densely packed plane.

On the other hand, if glide actually does take place on the most densely packed plane (shown above to be $\{1 \overline{1}\})$, then the measurements recorded as satisfying $\{100\}$ glide must correspond also with the geometrical requirements for the $\{1 \mathrm{I} T\}$ mode of glide, which will be a curious eoincidence.

Calculation shows that this coincidence actually exists, thus : (1) Double glide was observed on surfaces symmetrically disposed in relation to an axis of the mercury wire examined. The angle between the two planes was $74 \cdot 4^{\circ}$ which, corrected for elongation, gave $78 \cdot 8^{\circ}$ as the original dihedral angle. It was pointed out that the smaller dihedral angle between rhomb faces is $80 \cdot 3^{\circ}$, and this close correspondence was adduced as evidence for glide on rhomb faces. Calculation shows, however, that the dihedral angle between two $\{11 \overline{1}\}$ planes is $75 \cdot 5^{\circ}$, so that this particular observation may well be considered as evidence for glide on $\{11 \overline{1}\}$ planes. Andrade and Hutchings reported having measured several cases of double glide, in every case finding the corrected included angle to be slightly less than $80.3^{\circ}$.

(2) Several measurements were taken of the angle between the slip lines and the twinning plane $\{110\}$. The measured angles varied between $75^{\circ}$ and $77.5^{\circ}$. The calculated angle being $77 \cdot 4^{\circ}$, the correspondence was held as additional confirmation of $\{100\}$ glide. However, calculation shows that the angle between the $\{110\}$ (twinning plane) and either of two $\{11 \overline{1}\}$ pyramidal planes is $82 \cdot 3^{\circ}$, which appears to be sufficiently near the observed angles to be considered as evidence for $\{11 \overline{1}\}$ glide. The crystal in which the value of $75^{\circ}$ was obtained was stated by Andrade

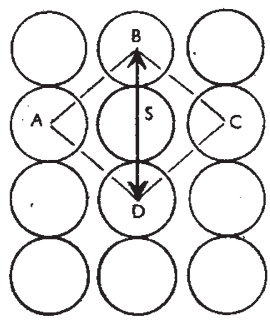

$\{100\}$ packing

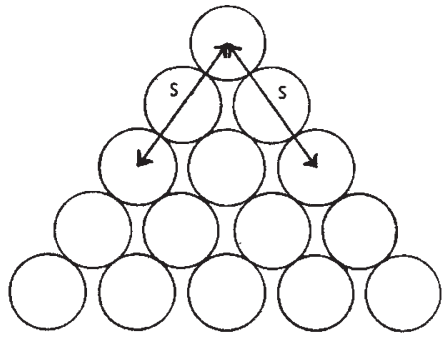

\{11î packing
COMPARISON OF ATOMIC PACKING ON DIFFERENT PLANES OF SINGLE MERCURY CRYSTAL.

and Hutchings to be not very favourable for measurement.

(3) The third set of measurements were those made on a wire extended to rupture. Traces of glide on the surface of rupture were observed to make an angle of almost $50^{\circ}$ with a line of commencing rupture parallel to the long diagonal of a rhomb face, and this is stated to agree with the value of $49 \cdot 1^{\circ}$ to be expected if the glide direction is a short diagonal of the rhomb face. Calculation shows that traces of $\{100\}$ planes on the adjacent rhomb faces make an angle of $40.9^{\circ}$, with lines parallel to the long diagonal. This is $9^{\circ}$ different from the observed angle. If the line of commencing rupture seen was unmis. takably parallel to the long diagonal, traces of a $\{1 \mathrm{I} \overline{1}\}$ plane on a twinning plane $\{110\}$ could make with that line an angle of $52.9^{\circ}$, which may be considered sufficiently near $50^{\circ}$ (observed) to be held as confirmation of $\{11 \overline{1}\}$ glide. This then requires $(a)$ the observed surface of fracture to be a twinning plane in order to explain the existence of the long diagonal as a commencing line of rupture, and $(b)$, the final fracture by direct separation. It is suggested this is a probable mode of fracture at the part of the crystal concerned.

With regard to the glide direction, the evidence remains in favour of this being the short diagonal of the rhomb face, while in regard to the twinning plane, no contradiction is involved in accepting the evidence for $\{110\}$.

Summing up, it would appear that : (a) the $\{11 \overline{1}\}$ planes are those most closely packed; $(b)$ glide takes place on these planes ; $(c)$ the direction of glide is the short diagonal of the rhomb face; $(d)$ there are two such glide directions in each glide plane.

Magnesium Elektron, Ltd., A. Fisher. Clifton Junction, Manchester.

1 Andrade, E. N. da C., and Hutchings, P. J., Proc. Roy. Soc., A, 148, 120. (1935).

Mr. Frsher is, I expect, right when he points out that the $\{11 \overline{1}\}$ planes are the most closely packed in a mercury single crystal, although I have not the time to check the work. The experiments to which he refers were carried out some years ago, and all notes referring to them were destroyed with my laboratory. Whether he is right in his surmise that the $\{1 \bar{I}\}$ planes may be the glide planes I cannot say. The only way of settling the question satis. factorily is by the X-ray method. I always intended to get the attribution checked in this way, but at the time when the work was done an X-ray set suitable for this purpose had not been installed, as it 